\title{
Behavioral CRs elicited by a lithium- or an amphetamine-paired contextual test chamber
}

\author{
LINDA A. PARKER, KAREN HILLS, and KRISTA JENSEN \\ University of New Brunswick, Saint John, New Brunswick, Canada
}

\begin{abstract}
The behavioral CRs elicited by a drug-paired contextual cue in rats were measured in a series of experiments. The first three experiments measured the pattern of CRs elicited by a lithiumand an amphetamine-paired CS chamber. The final experiment measured the pattern of URs elicited by lithium or amphetamine when administered in the same chamber. The suppression of grooming-related activities (body washing, face washing, and/or scratching) appeared as a nonspecific effect of both drug USs that was capable of becoming conditioned to the contextual chamber cue. A number of other behaviors differed among conditions, which provides evidence that contextual cue-drug associations are drug-specific. Of the behaviors that differed between the two US drug conditions, lithium CRs showed evidence of behavioral activation (enhanced rearing and limb flicking), but amphetamine CRs showed evidence of behavioral suppression (line crossing, rearing, and shaking). The UR patterns shared some similar and some dissimilar elements of the CR patterns elicited by the CS chamber for both lithium and amphetamine; in fact, by the activity and rearing measures, the URs and CRs were opposite in direction. The results exclusively supported neither stimulus substitution nor conditioned compensatory response accounts of Pavlovian conditioning; instead, each process appears to have accounted for different components of the overall CR pattern.
\end{abstract}

Conditioned drug effects were originally described by Pavlov (1927). A dog was presented a tone, the conditioned stimulus (CS) that was paired with an injection of apomorphine, the unconditioned stimulus (US). After a number of such pairings, the tone elicited the behavioral symptoms of the drug state; that is, the tone elicited conditioned responses (CRs) that were similar in nature to the unconditioned responses (URs) produced by the drug. Since Pavlov's original investigations, conditioned drug effects have been shown with a variety of pharmacological agents. For instance, Pickens and Crowder (1967) demonstrated that the increase in activity produced by amphetamine could be conditioned to an environmental chamber when the chamber was paired with amphetamine on each of six trials.

Considerable research effort has been invested in determining how drug states change a rat's preference for a flavor or an environmental CS (see Riley \& Baril, 1976), but little has been invested in determining the pattern of behavioral responses elicited by that drugpaired CS. The following series of experiments measured the conditioned responses (CRs) elicited by a contextual test chamber that was previously paired with either

The authors wish to thank Robert Smith and Lee Wilson for technical assistance in conducting Experiment 4 and scoring various tapes. This research was supported by the Natural Sciences and Engineering Research Council of Canada, Grant A7464, as well as by the UNB Research Fund. Send reprint requests to: Linda A. Parker, P.O. Box 5050, Division of Social Sciences, University of New Brunswick, Saint John, N.B., Canada E2L 4L5. lithium (Experiments 1 and 2) or amphetamine (Experiment 3). These experiments were meant to characterize the nature of the behavioral responses that occur when rats are placed in a distinctive location that has previously been paired with a drug state. By employing a similar procedure in Experiments 1, 2, and 3, we were able to compare lithium CRs with amphetamine CRs. Parker (1982) recently reported that when flavors serve as a CS, both lithium and amphetamine support the CRs of enhanced general activity and rearing; however, only lithium supported the CR of chin rubbing. The finding of a lithium-specific CR suggested that different UR mechanisms are involved in flavor-lithium and flavor-amphetamine associations. The following series of experiments were undertaken to determine whether different UR mechanisms were also involved in test chamber-lithium and test chamber-amphetamine associations. The various behaviors observed were previously reported by Parker (1982) and are presented in Table 1. The final experiment in the series measured the URs elicited by lithium and amphetamine to determine whether the CRs were similar or dissimilar to the URs.

\section{EXPERIMENT 1}

Experiment 1 measured various behavioral responses elicited by a chamber CS that had been paired on three occasions (every other day) with one of three doses of $.15 \mathrm{M}$ lithium chloride $(0.3,1.2$, or $3.0 \mathrm{mEq} / \mathrm{kg})$. To control for sensitization effects, noncontingently in. jected control groups were injected with physiological 
Table 1

Definition of Behavioral Categories

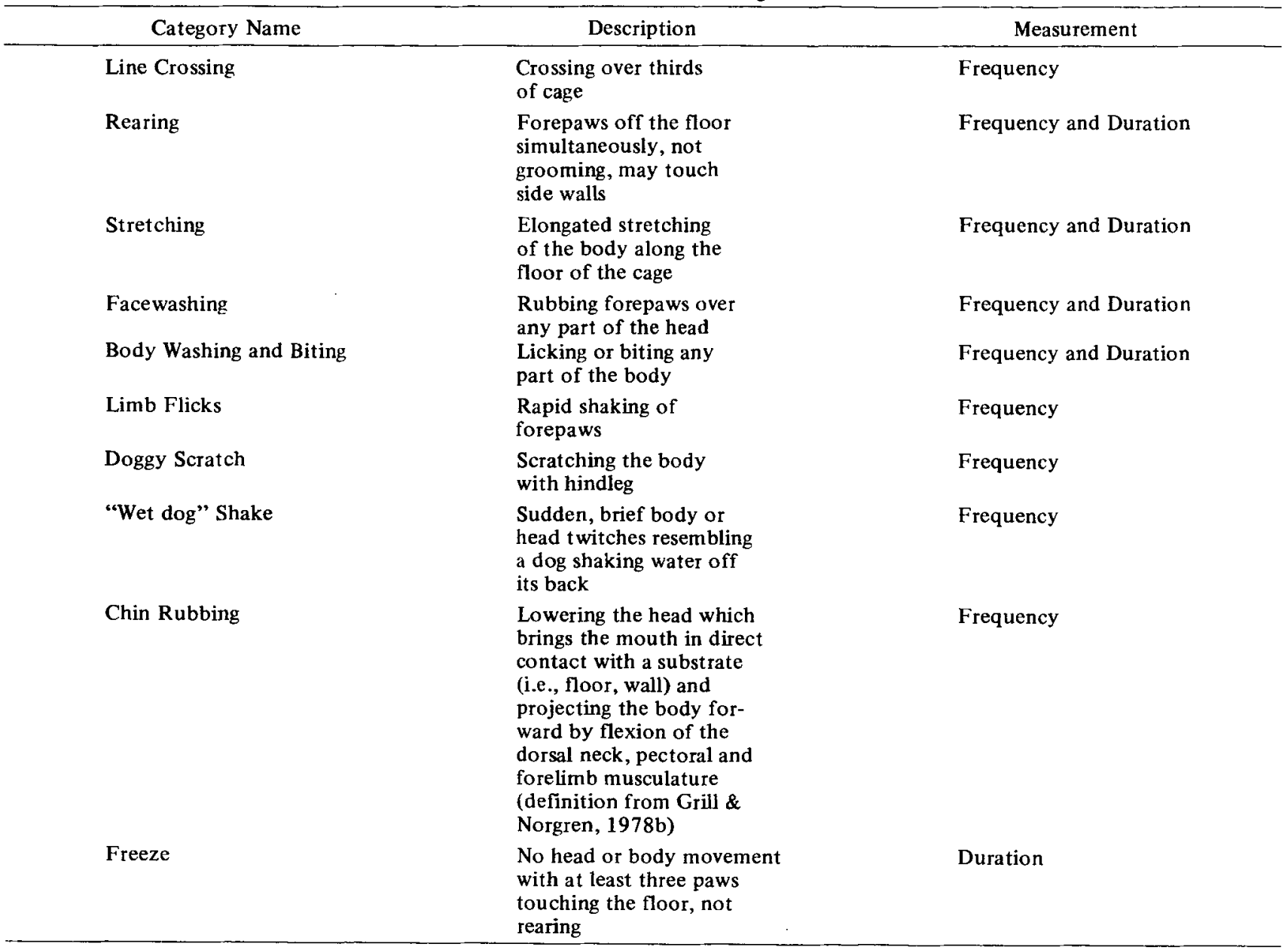

saline on "conditioning" days and lithium chloride on adjacent days.

\section{Method}

\section{Subjects}

The subjects were 42 male Sprague-Dawley rats weighing between 279 and $380 \mathrm{~g}$ on the first conditioning day and maintained on ad-lib Purina Rat Chow and water throughout the experiment. They were housed individually in wire-mesh cages and maintained on a $12-/ 12-\mathrm{h}$ (light/dark) schedule of illumination.

\section{Apparatus}

The conditioning chamber $(45 \times 25 \times 20 \mathrm{~cm})$ was constructed of glass and had a wire-mesh floor and ceiling. The room was illuminated by two $25-W$ light bulbs placed $30 \mathrm{~cm}$ from either side of the chamber. A mirror was placed behind the chamber at an angle to facilitate observation of the rats while they were in the chamber.

The behavior of each rat while in the chamber was recorded on videotape. A Hitachi HV -62 videocamera, which was focused on the chamber, transmitted the rats' image through a video- cassette recorder (JVC-CR 6060U), located in the adjacent toom, to a 12-in. Electrohome monitor. A 20-channel EsterlineAngus event recorder was used to record the frequency and duration of the various behaviors described in Table 1 .

\section{Procedure}

Conditioning trials. The rats received three conditioning trials. On each trial, each rat was injected with either lithium chloride (LiCl) or saline, $5 \mathrm{~min}$ before being placed in the CS chamber for $15 \mathrm{~min}$. The six groups $(\mathrm{n}=7)$ differed according to whether $\mathrm{LiCl}$ was administered contingently (CS+ groups) or noncontingently (CSc groups) upon placement in the CS chamber and by the dose of $.15 \mathrm{M} \mathrm{LiCl}(0.3,1.2$, or $3.0 \mathrm{mEq} / \mathrm{kg})$ that was administered.

The conditioning trials occurred on Monday, Wednesday, and Friday. Twenty-four hours after each conditioning trial, the rats in the CSc groups were injected with $0.3,1.2$, or $3.0 \mathrm{mEq} / \mathrm{kg}$ of $.15 \mathrm{M} \mathrm{LiCl}$ and then returned to their home cage. On the same day, the rats in the CS+ groups were injected with 20,8 , or $2 \mathrm{ml} / \mathrm{kg}$ of physiological saline, depending upon the dose of $\mathrm{LiCl}$ with which they had been conditioned.

Test trial. Three days after the final conditioning trial, the rats were tested for conditioned behavioral responses elicited by the CS chamber. Each rat was placed in the CS chamber for 
$15 \mathrm{~min}$ and its behavior was videotaped. An observer, blind to the experimental conditions, scored the videotaped records, using a 20-channel Esterline-Angus event recorder, according to the categories listed in Table 1. The 15-min scores of the rats in each behavioral category were divided into three 5 -min intervals. These scores were then analyzed in $2 \times 3 \times 3$ ANOVAs with the factors of CS condition (CS+, CSc), US condition $(0.3,1.2$, and $3.0 \mathrm{mEq} / \mathrm{kg}$ of $.15 \mathrm{M} \mathrm{LiCl})$, and 5 -min intervals of testing $(5,10$, and $15 \mathrm{~min})$.

\section{Results}

The following behavioral categories revealed evidence of lithium-induced conditioned drug effects: rearing (duration), body wash (frequency and duration), limb flick (frequency), and scratch (frequency). Each of these behaviors is presented in Figure 1 and will be discussed separately.

\section{Rearing}

The mean duration of rearing behavior in the $15-\mathrm{min}$ test of each group is presented in the upper panel of Figure 1 . The $2 \times 3 \times 3$ ANOVA for the rearing duration scores revealed a significant effect of CS condition $[F(1,36)=4.6, p<.05] ;$ overall, the $\mathrm{CS}+$ groups showed longer periods of rearing than the CSc groups. There was also a significant $C S$ condition $X$ US condition $X$ intervals interaction $[F(4,72)=2.5, p<.05]$. Subsequent $2 \times 3$ repeated measures ANOVAs for each US dose level revealed significant effects only at the $3.0-\mathrm{mEq} / \mathrm{kg}$ dosage level. At the $3.0-\mathrm{mEq} / \mathrm{kg}$ dose level, the CS+ group reared for a longer duration overall than did the CSc group $[F(1,12)=9.6, p<.01]$. The analysis also revealed a significant $C S$ condition $X$ intervals interaction $[F(2,24)=3.8, p<.05]$; by Newman-Keuls tests, at the $3.0-\mathrm{mEq} / \mathrm{kg}$ dose level, Group CS+ showed longer periods of rearing than Group CSc only during the second 5-min interval of testing. No other effects were significant.

\section{Body Washing}

The second panel in Figure 1 presents the mean duration of body washing by the various groups in the 15 -min test of Experiment 1. The $2 \times 3 \times 3$ ANOVAs revealed that the rats in the CS+ groups showed fewer bouts of body washing $[\mathrm{F}(1,36)=4.9, \mathrm{p}<.05]$ and spent less time body washing $[\mathrm{F}(1,36)=6.9, \mathrm{p}<.05]$ than the rats in the CSc groups. No other effects were significant.

\section{Limb Flicking}

The third panel of Figure 1 presents the mean frequency of limb flicks during the test. As is evident, the CS+ groups showed a greater number of limb flicks in the 15 -min test period than did the CSc groups $[F(1,36)$ $=10.3, \mathrm{p}<.01]$, regardless of US condition.

\section{Scratching}

The mean frequency of scratching in each group is presented in the final panel of Figure 1. The CS+ groups

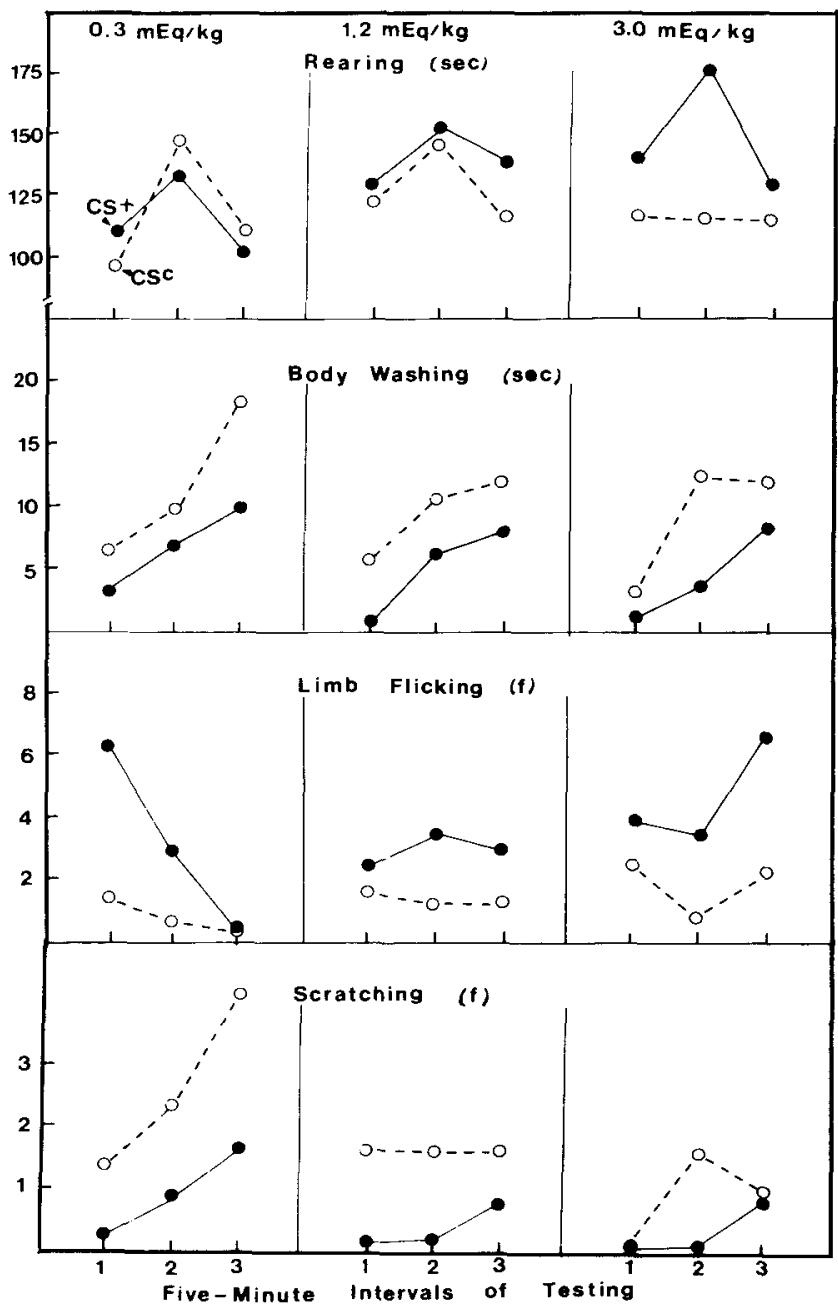

Figure 1. Mean frequency (f) or duration (sec), during each 5-min interval of testing, of each behavior that showed evidence of conditioning after three conditioning trials in Experiment 1. The various groups were conditioned with $0.3,1.2$, or $3.0 \mathrm{mEq} / \mathrm{kg}$ of $\mathrm{LiCl}$. The CS+ groups are depicted with closed circles and solid lines; the CSc groups are depicted with open circles and broken lines.

showed less scratching behavior than did the CSc groups $[F(1,36)=4.0, p<.05]$. No other effects were significant.

\section{Discussion}

When exposed to a CS chamber that had been previously paired on three occasions with $\mathrm{LiCl}$, rats showed a suppressed frequency and duration of body-washing activity and a suppressed frequency of scratching relative to control rats which had received noncontingent injections of $\mathrm{LiCl}$. On the other hand, the CS+ groups showed a greater number of limb flicks than did the CSc groups. The duration of rearing activity was also affected by the experimental manipulations. At the highest 
dosage level $(3.0 \mathrm{mEq} / \mathrm{kg})$, the CS+ group spent more time rearing than did the $\mathrm{CSc}$ group.

\section{EXPERIMENT 2}

After three pairings of a test chamber and lithium in Experiment 1, rats showed evidence of lithium-induced conditioned behavioral effects. Experiment 2 was designed to determine whether a single conditioning trial would be sufficient to produce a conditioned behavioral change to the $C S$ chamber paired with $3.0 \mathrm{mEq} / \mathrm{kg}$ of $.15 \mathrm{M} \mathrm{LiCl}$, the highest dose used in the previous experiment.

\section{Method}

The subjects were 14 male Sprague-Dawley rats weighing between 234 and $322 \mathrm{~g}$ on the conditioning day. On the conditioning day, each rat was injected with either $3.0 \mathrm{mEq} / \mathrm{kg}$ of $.15 \mathrm{M} \mathrm{LiCl}(\mathrm{n}=7)$ or $20 \mathrm{ml} / \mathrm{kg}$ of physiological saline $(\mathrm{n}=7)$ 5 min prior to being placed in the CS chamber. The rats remained in the chamber for $15 \mathrm{~min}$. The rats in the saline control group were also injected with $3.0 \mathrm{mEq} / \mathrm{kg}$ of $.15 \mathrm{M} \mathrm{LiCl} 24 \mathrm{~h}$ after the conditioning trial to equate the groups on the basis of prior experience with $\mathrm{LiCl}$.

Three days after the conditioning day, the rats were tested. Each rat was placed in the CS chamber for $15 \mathrm{~min}$ and its behavior was videotaped. The test day videotapes were scored by a blind observer for the various behaviors listed in Table 1. The scores of each behavioral category were analyzed in a $2 \times 3$ repeated measures ANOVA with the factors of CS condition and 5 -min intervals.

\section{Results and Discussion}

The behavioral category of scratching revealed evidence of conditioning after a single CS chamber-lithium pairing. As is evident in Figure 2, the $2 \times 3$ repeated measures ANOVA revealed a significant $\mathrm{CS}$ condition effect $[F(1,12)=4.6, p<.05]$. Across the 15 -min test period, Group CS+ showed less scratching than Group CSc. No other behaviors differed between the groups.

\section{EXPERIMENT 3}

In the previous two experiments, lithium chloride served as the US drug that was paired with a distinctive contextual test chamber. The results revealed that three pairings of the chamber and lithium elicited suppressed scratching and body washing, but enhanced limb flicking. Additionally, at the highest dosage level employed $(3.0 \mathrm{mEq} / \mathrm{kg})$, three conditioning trials produced the CR of enhanced rearing duration. A single pairing produced only the CR pattern of suppressed scratching.

Parker (1982) recently described a pattern of behavioral CRs that are elicited by a lithium-paired and an amphetamine-paired flavor CS. Although both drugs supported a CR pattern of enhanced general activity and rearing activity, only lithium supported a behavioral $C R$ of chin rubbing. The finding that a drug-specific CR was

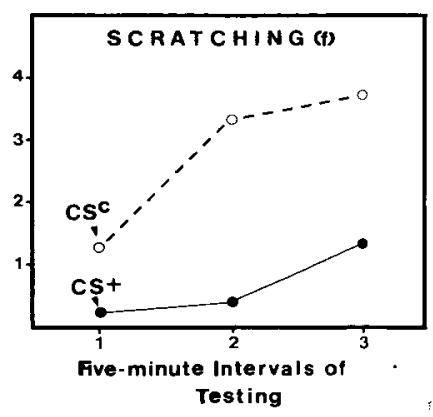

Figure 2. Mean frequency (f) of scratching behavior shown by the CS+ and the CSc groups during each 5 -min in terval of testing in Experiment 2 following a single conditioning trial with $3.0 \mathrm{mEq} / \mathrm{kg}$ of $\mathrm{LiCl}$.

elicited by the CS+ flavor indicates that different US elements are most likely associated with the CS flavor when the two drugs of lithium and amphetamine are employed as the US. If such is the case, then one must assume that the mechanism responsible for the development of a conditioned flavor aversion differs between these two US drugs, even though, in Parker's (1982) experiment, each drug produced an equivalent consummatory avoidance response to the CS+ flavor.

Experiment 3 employed amphetamine as the US agent in a paradigm similar to that of Experiments 1 and 2. Rats were administered either one or three CS chamberamphetamine pairings in a contingent or a noncontingent manner. The experiment determined whether the set of behavioral CRs elicited by an amphetaminepaired contextual test chamber was similar or dissimilar to the set of CRs elicited by the lithium-paired contextual test chamber in Experiments 1 and 2. That is, was there a common nonspecific US across drugs associated with the CS, as suggested in the literature of conditioned flavor aversion learning (e.g., Bravemen, 1975), or was the US associated with the CS specific to each drug, as suggested in the conditioned drug effects literature (e.g., Pickens \& Crowder, 1967)? The dose of amphetamine used $(3.0 \mathrm{mg} / \mathrm{kg})$ was selected because Parker (1982) had reported that this dose produces a flavor aversion equivalent to that produced by the highest dose of lithium employed in Experiment 1 $(3.0 \mathrm{mEq} / \mathrm{kg})$. Therefore, by the flavor-aversion measure, the two doses produce quantitatively similar associative effects.

\section{Method}

The subjects were 28 male Sprague-Dawley rats weighing between 233 and $273 \mathrm{~g}$ on Conditioning Day 1. There were four groups $(\mathrm{n}=7)$ of rats: Group CS+-3, Group CSc-3, Group CS+-1, and Group CSc-1. On each of three conditioning trials, the rats in Group CSt-3 were injected with $3 \mathrm{mg} / \mathrm{kg}$ of d-amphetamine in solution with saline $5 \mathrm{~min}$ before being placed in the CS chamber for $15 \mathrm{~min}$. The conditioning trials occurred on Monday, Wednesday, and Friday. On the same three conditioning days, the rats in Group CSc-3 were injected with $18 \mathrm{ml} / \mathrm{kg}$ 
of physiological saline $5 \mathrm{~min}$ before being placed in the CS chamber for $15 \mathrm{~min}$. Twenty-four hours after each conditioning trial, the rats in Group CSc-3 received a noncontingent injection of amphetamine and the rats in Group CS+-3 received an injection of saline. The rats in Groups CS+-1 and CSc-1 were given a single conditioning trial on Friday. Group CS +1 received $3 \mathrm{mg} / \mathrm{kg}$ of amphetamine and Group CSc-1 received $18 \mathrm{ml} / \mathrm{kg}$ of saline $5 \mathrm{~min}$ prior to placement in the CS chamber for $15 \mathrm{~min}$. Twenty-four hours later, the rats in Group CSc-1 were injected with $3 \mathrm{mg} / \mathrm{kg}$ of amphetamine and the rats in Group CSc-1 were injected with phy siological saline.

The test trial occurred 3 days after the final conditioning trial. The procedure was identical to that employed in Experiment 1 . For purposes of scoring the tapes, the 15 -min test was divided into three 5-min intervals. The scores of the rats' behavior in each category were analyzed in $2 \times 2 \times 3$ ANOVAs with the factors of $\mathrm{CS}$ condition (CS+, CSc), number of conditioning trials $(1,3)$, and 5 -min intervals $(5,10$, and $15 \mathrm{~min})$.

\section{Results}

Figure 3 presents the behaviors, measured in Experiment 3 , which showed evidence of becoming conditioned to the amphetamine-paired chamber CS. Each of these behaviors will be discussed separately.

\section{Line Crossings}

The $2 \times 2 \times 3$ repeated measures ANOVA of the line-crossing scores presented in Figure 3 revealed a significant $C S$ condition $X$ intervals interaction $[F(2,48)$ $=4.8, \mathrm{p}<.025]$. Subsequent Newman-Keuls comparisons of the CS condition at each interval indicated that the pooled CS+ groups (pooled across one and three conditioning trials) showed less line crossing than the pooled CSc groups during the first 5 -min interval of testing $(\mathrm{p}<.05)$ but not during the last two 5 -min intervals of testing.

\section{Rearing}

Analysis of the rearing-frequency data presented in Figure 3 revealed a significant $C S$ condition $X$ intervals interaction $[F(2,48)=6.1, p<.01]$. A subsequent Newman-Keuls analysis of the CS condition at each interval revealed that the pooled $\mathrm{CS}+$ groups reared less frequently than the pooled CSc groups during the first 5 -min interval of testing ( $\mathrm{p}<.05)$, but not during the later test intervals.

\section{Face Washing}

Although only the face-wash-duration measure is depicted in Figure 3, both the frequency and duration of face washing were suppressed in the CS+ groups relative to the CSc groups, regardless of the interval of testing or the number of conditioning trials. This is verified by a significant $\mathrm{CS}$ condition effect for both the frequency measure $[F(1,24)=7.5, p<.01]$ and the duration measure $[F(1,24)=10.4, p<.01]$. No other effects were significant.

\section{Body Washing}

The mean duration of body washing is depicted in Figure 3. The $2 \times 2 \times 3$ repeated measures ANOVA

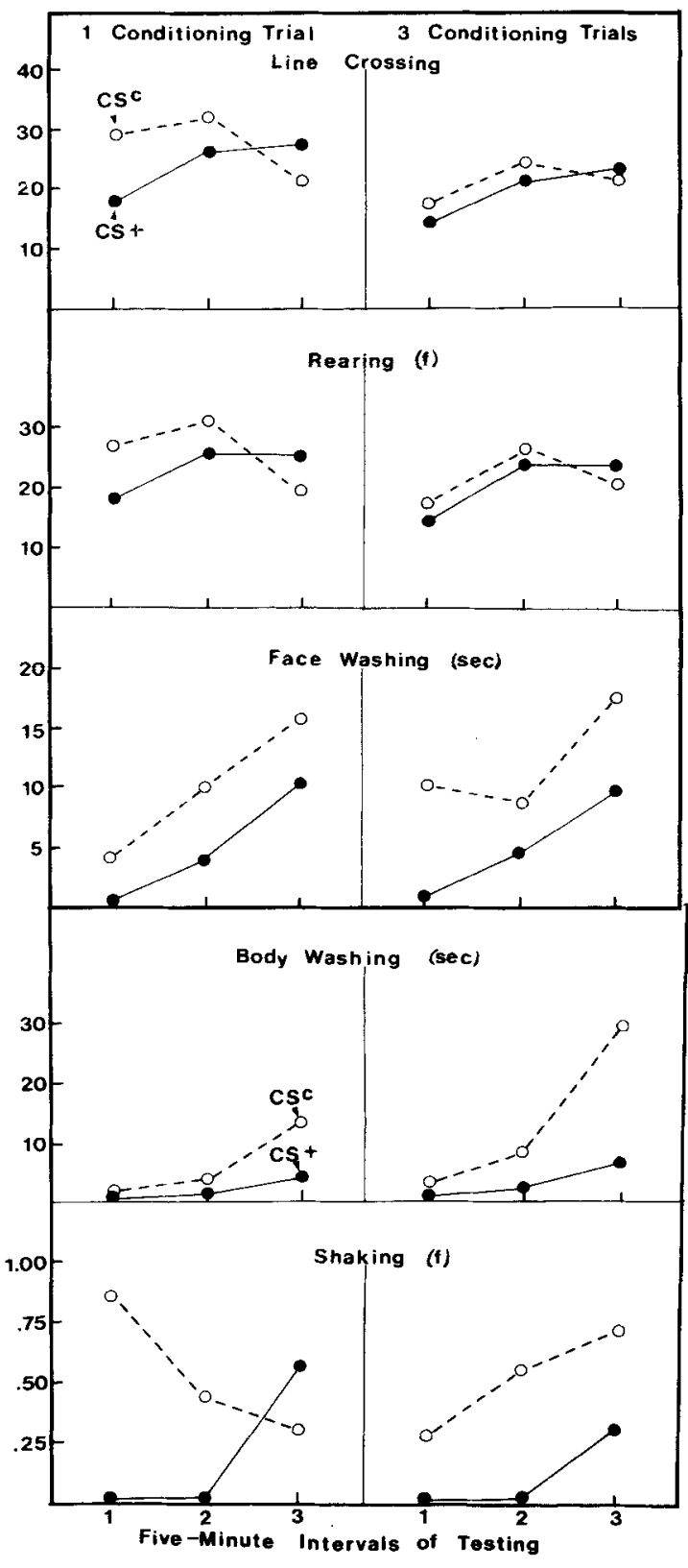

Figure 3. Mean frequency (f) or duration (sec), during each 5-min interval of testing, of each behavior that showed evidence of conditioning in Experiment 3. The groups depicted in the left-hand sections were given one conditioning trial with $3 \mathrm{mg} / \mathrm{kg}$ of d-amphetamine; the groups depicted in the right-hand section were given three conditioning trials with $3 \mathrm{mg} / \mathrm{kg}$ of d-amphetamine. The CS+ groups are depicted with closed circles and solid lines; the CSc groups are depicted with open circles and broken lines.

revealed a significant $C S$ condition effect $[F(1,24)=$ $9.7, \mathrm{p}<.01]$; overall, the CS+ groups spent less time body washing than did the CSc groups. There was also a significant $C S$ condition $X$ intervals interaction $[F(2,48)=5.6, p<.01]$. Subsequent Newman-Keuls tests between the pooled CS+ groups and the pooled 
CSc groups at each interval revealed that the difference was significant only during the last 5 -min interval of testing $(\mathrm{p}<.05)$.

\section{Shaking}

The final panel in Figure 3 represents the mean frequency of "wet dog" shakes by each group during the test. The analysis revealed an overall CS condition effect $[F(1,24)=6.7, p<.025]$. The CS+ groups showed fewer "wet dog" shakes than did the CSc groups.

\section{Discussion}

A number of behaviors revealed evidence of becoming conditioned to an amphetamine-paired contextual chamber CS. The general behavioral pattern demonstrated by rats exposed to the CS chamber was suppression of behavior. The CS+ groups showed suppressed body washing, face washing, and shaking over the test period. Additionally, during the first 5-min interval of testing, the CS+ groups tended to cross fewer lines and to rear less frequently than did the CSc groups. Each of the CRs reported was observed following either one or three conditioning trials.

When compared with the pattern of behaviors elicited by a lithium-paired CS chamber, similarities and dissimilarities are apparent. The grooming-related behaviors of body washing, face washing, and scratching were suppressed in the presence of either a lithium-paired or an amphetamine-paired contextual chamber CS. A lithium. paired context suppressed body washing and scratching, and an amphetamine-paired context suppressed face washing and body washing. Therefore, a common CR pattern of suppressed grooming is supported by either US drug. On the other hand, some behavioral CRs differed between the two US drug conditions. Lithium supported the CRs of enhanced limb flicking and rearing (at the highest dosage level), whereas amphetamine supported the CRs of suppressed shaking overall and suppressed line crossings and rearing during the first $5 \mathrm{~min}$ of testing. Thus, of the behaviors that differed between the two US drug conditions, lithium CRs showed evidence of behavioral activation, whereas the amphetamine CRs were evidenced as behavioral suppression. Since not all CRs are similar between the US drug conditions of lithium and amphetamine, our results support the contention that the US elements associated with the contextual chamber cue differ between the drugs of lithium and amphetamine; that is, chamberdrug associations are drug specific.

The results of Experiment 3 indicate that an amphetamine-associated CS chamber elicits a pattern of generally suppressed activity. However, Pickens and Crowder (1967) and Tilson and Rech (1973) have reported that exposure to amphetamine-associated cues produce enhanced activity, much like the unconditioned drug effect. There were quite a few parametric differences between our experiment and those of Pickens and Crowder (1967) and Tilson and Rech (1973). The prior experiments employed a larger number of conditioning trials and lower doses of amphetamine (0.5. $1.8 \mathrm{mg} / \mathrm{kg}$ ) than those used in the present experiment; either or both of these factors may account for the difference in the pattern of results. Additionally, both prior studies employed a CS preexposure paradigm in which the rats were exposed to the CS chamber on a number of occasions prior to the conditioning trials. Since preexposure to a CS reduces its efficacy in becoming associated with the US (e.g., Lubow \& Moore, 1959), the prior habituation training employed by Pickens and Crowder (1967) and Tilson and Rech (1973) may have retarded the development of the CRs reported in the present experiment.

\section{EXPERIMENT 4}

The CRs elicited by an amphetamine-paired CS chamber were seen as a general suppression of various behaviors; however, amphetamine is a behavioral stimulant that enhances general activity levels. The CRs elicited by a lithium-paired CS chamber were mixed with some behavioral stimulation and some behavioral suppression. Lithium chloride is generally reported to act as a suppressor of general activity (e.g., Johnson, 1979).

The purpose of Experiment 4 was to investigate the nature of the URs that are elicited by lithium and amphetamine in a situation similar to that in which lithium and amphetamine CRs were measured in the previous three experiments. The lithium test groups and the amphetamine test groups were run 1 week apart; however, they will be presented together in the method section as a single experiment.

\section{Method}

Forty male Sprague-Dawley rats between 267 and $395 \mathrm{~g}$ were maintained on ad-lib food and water throughout the experiment. On each of three consecutive adaptation days, each tat was placed in the CS chamber (described in Experiment 1) for $30 \mathrm{~min}$. Twenty-four hours after the final adaptation trial, the rats were tested. Each rat was injected with the appropriate solution and 2 min later was placed in the CS chamber for $30 \mathrm{~min}$. The behavior of the rats during the test trial was videotaped.

The groups given the lithium test were run during the first week and the groups given the amphetamine test were run 1 week later. For the lithium test trial, 20 rats were randomly divided into two groups: Group $\mathrm{LiCl}(\mathrm{n}=10)$ and Group Saline $(n=10)$. On the test day, Group $\mathrm{LiCl}$ was injected with $3.0 \mathrm{mEq} / \mathrm{kg}$ of $.15 \mathrm{M} \mathrm{LiCl}$ and Group Saline was injected with $20 \mathrm{ml} / \mathrm{kg}$ of physiological saline.

For the amphetamine test trial, the remaining 20 rats were randomly divided into Group Amphetamine $(n=10)$ and Group Saline $(n=10)$. The amphetamine injection consisted of $3 \mathrm{mg} / \mathrm{kg}$ of d-amphetamine in solution with physiological saline $(1 \mathrm{mg}$ d-amphetamine $/ 6 \mathrm{ml}$ saline) and the saline injection consisted of $18 \mathrm{ml} / \mathrm{kg}$ of physiological saiine. 
The videotaped records were scored by an observer who was blind to the experimental conditions. For the data analysis, the $30-\mathrm{min}$ test period was divided into 103 -min intervals. The lithium test and the amphetamine test were analyzed separately. The data for each of the behaviors described in Table 1 were then analyzed in a $2 \times 10$ repeated measures ANOVA with the factors of experimental condition (drug/saline) and 3-min intervals (3-30 $\mathrm{min})$. Two additional behaviors were observed in the lithium UR data which are not included in Table 1 . The first behavior is body dragging, defined as elongation of the body with the belly pulled along the floor of the cage by the front paws. The second behavior is lying on belly, defined as lying on the belly in the cage. This behavior is different from freezing because it usually includes movement of another part of the body (i.e., the head). These behaviors were analyzed in the same manner as those described in Table 1.

\section{Results}

The behaviors that differed between drug-injected and saline-injected rats are presented in Figures 4 and 5. In Figure 4, the data presented in the left-hand sections represent the lithium test, and the data presented in the right-hand sections represent the amphetamine test. The solid lines represent the drug condition (either Group $\mathrm{LiCl}$ or Group Amphetamine), and the broken lines represent the saline condition. Each of the behaviors depicted in these figures will be discussed separately.

\section{Line Cross}

Panel 1 in Figure 4 presents the line crossing data for the lithium test and for the amphetamine test. The $2 \times 10$ repeated measures ANOVA for the lithium test revealed a significant experimental condition effect $[F(1,18)=36.8, p<.01]$; Group $\mathrm{LiCl}$ showed less linecrossing activity than did Group Saline. In addition, a significant experimental condition $X$ intervals interaction $[F(9,162)=3.5, p<.01]$ was the result of Group LiCl's showing suppressed line-crossing activity during Min 3-21 (ps < .05), but not during Min 24-30.

The amphetamine test data also revealed a significant experimental condition effect $[F(1,18)=37.7, p<.01]$ and a significant experimental condition $X$ intervals interaction $[F(9,162)=4.7, p<.01]$. The groups did not differ during Min 3-15; however, Group Amphetamine showed enhanced line-crossing activity during Min 18-30 (ps < .05). Thus, during the 30-min test, Group $\mathrm{LiCl}$ showed suppressed line-crossing activity during the first $21 \mathrm{~min}$ of testing and Group Amphetamine showed enhanced line-crossing activity during the last $15 \mathrm{~min}$ of testing.

\section{Rearing}

Panels 2 and 3 in Figure 4 present the rearing data: Panel 2 presents rearing frequency and panel 3 presents rearing duration. For the lithium test, both measures revealed a significant experimental condition effect [frequency, $F(1,18)=43.2, \mathrm{p}<.01 ;$ duration, $\mathrm{F}(1,18)=$ $17.3, \mathrm{p}<.01]$ and a significant experimental condition $X$ intervals interaction [frequency, $F(9,162)=31.4$,

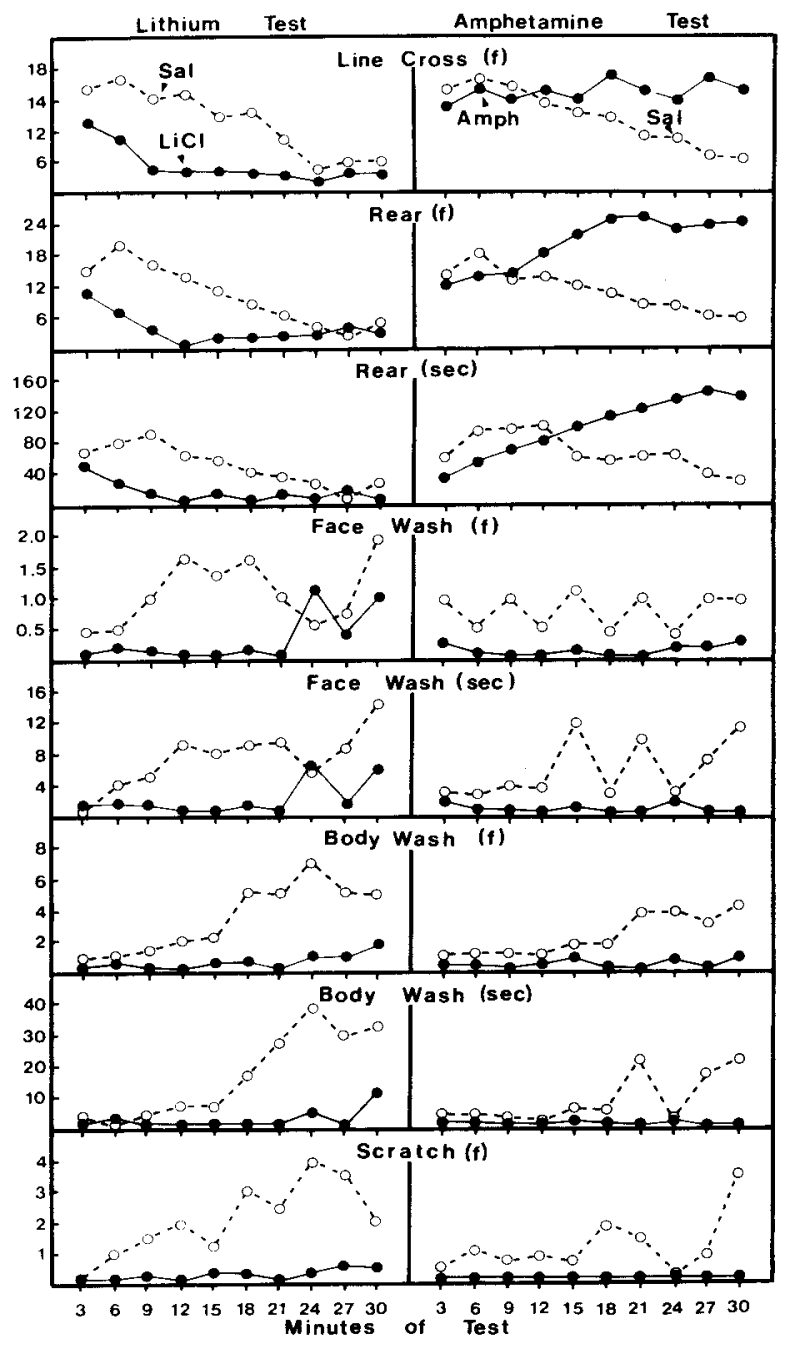

Figure 4. Mean frequency (f) and duration (sec) of various behaviors elicited by either $3.0 \mathrm{mEq} / \mathrm{kg}$ of lithium chloride (left-hand sections) or $3 \mathrm{mg} / \mathrm{kg}$ of d-amphetamine (right-hand sections) across 3-min intervals of testing in Experiment 4. The US drug groups are depicted with closed circles and solid lines; the saline control groups are depicted with open circles and broken lines.

$\mathrm{p}<.01$; duration, $\mathrm{F}(9,162)=16.7, \mathrm{p}<.01]$. Group LiCl showed a suppressed frequency and duration of rearing relative to Group Saline during Min 3-21 (ps $<.05$ ), but the groups did not differ during Min 24-30.

For the amphetamine test, both measures of rearing also revealed a significant experimental condition effect [frequency, $F(1,18)=13.6, \mathrm{p}<.01 ;$ duration, $\mathrm{F}(1,18)=$ $8.0, p<.05]$ and a significant experimental condition $X$ intervals interaction [frequency, $F(9,162)=9.2, p<$ .01 ; duration, $F(9,162)=25.5, \mathrm{p}<.01]$. Although the groups did not differ during Min 3-21, Group Amphetamine reared more frequently than Group Saline during Min 15-30 (ps < .05). On the other hand, during Min 3-6, Group Amphetamine reared for a shorter dura- 


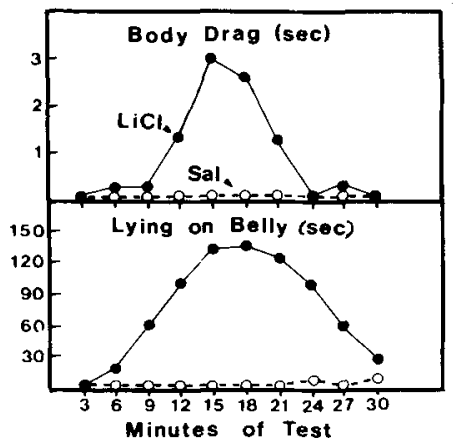

Figure 5. Mean duration of body dragging and lying on bellies shown by groups administered either $\mathrm{LiCl}$ or saline in Experiment 4 across 3 -min intervals of testing.

tion than did Group Saline ( $p s<.05$ ), and during Min 15-30 Group Amphetamine reared for a longer duration than did Group Saline.

The pattern of rearing behavior differed for the lithium and the amphetamine test. Group $\mathrm{LiCl}$ showed suppressed rearing activity for the first $21 \mathrm{~min}$ of testing. Group Amphetamine showed enhanced rearing activity during the last 15 min of testing.

\section{Face Washing}

The mean frequency and duration of face washing is presented in panels 4 and 5 of Figure 4. The lithium test revealed a significant experimental condition effect for face-wash frequency $[F(1,18)=22.8, p<.01]$ and for face-wash duration $[F(1,18)=21.8, \mathrm{p}<.01]$. For both measures, Group LiCl showed suppressed facewashing activity relative to Group Saline.

A pattern of results similar to the lithium test was apparent in the amphetamine test. A significant experimental condition effect was evident for face-wash frequency $[F(1,18)=22.0, p<.01]$ and for face-wash duration $[F(1,18)=30.5, p<.01]$. For both measures, Group Amphetamine showed suppressed face-washing relative to Group Saline.

\section{Body Washing}

Panels 6 and 7 of Figure 4 present the mean frequency and duration of body washing in Experiment 4. The lithium test revealed a significant experimental condition effect for body-wash frequency $[F(1,18)=$ 14.6, $\mathrm{p}<.01]$ and for body-wash duration $[\mathrm{F}(1,18)=$ $10.7, \mathrm{p}<.01]$. Additionally, the analysis revealed a significant experimental condition $X$ intervals interaction for frequency $[F(9,162)=4.3, p<.01]$ and for duration $[F(9,162)=3.4, p<.01]$. Group $\mathrm{LiCl}$ showed less body washing than did Group Saline during Min 9-30 (ps $<.05)$ and a shorter duration of body washing than did Group Saline during Min 18-30 (ps < .05).

The amphetamine test results also revealed a significant experimental condition effect for body-wash frequency $[F(1,18)=6.2, p<.05]$ and for body-wash duration $[F(1,18)=8.3, p<.01]$. The experimental condition $X$ intervals interaction was also significant for body-wash frequency $[F(9,162)=2.2, p<.05]$ and for body-wash duration $[F(9,162)=4.2, p<.01]$. Group Amphetamine showed fewer bouts of body wash. ing than did Group Saline during Min 21-30 (ps $<.05$ ) as well as a shorter duration of body washing during Min 21 and Min 27-30 (ps < .05). Thus, the pattern of body-washing activity was similar for the lithium and the amphetamine tests; both drugs produced suppression of body washing.

\section{Scratching}

The mean frequency of scratching is presented in panel 8 of Figure 4 . For both the lithium and the amphetamine test, the only significant effect was the experimental condition [lithium test, $F(1,18)=10.4$, $\mathrm{p}<.01$; amphetamine test, $\mathrm{F}(1,18)=6.3, \mathrm{p}<.05]$. Both Group $\mathrm{LiCl}$ and Group Amphetamine showed less scratching than did their corresponding Group Saline.

\section{Body Dragging and Lying on Belly}

Two additional behaviors were observed in Group $\mathrm{LiCl}$ that were never observed in Group Amphetamine. These two behaviors are presented in Figure 5. The mean duration of body dragging is depicted in the upper panel of Figure 5. A significant experimental condition effect $[F(1,18)=9.1, p<.01]$ and experimental condition $X$ intervals interaction $[F(9,162)=5.0, p<.01]$ was evident. Group $\mathrm{LiCl}$ engaged in the behavior of body dragging for a greater portion of the test period during Min 12-21 than did Group Saline (ps <.05).

The mean duration of lying on the belly is presented in the lower panel of Figure 5. The ANOVA revealed a significant experimental condition effect $[F(1,18)=$ $29.7, \mathrm{p}<.01]$ and a significant experimental condition $X$ intervals interaction $[F(9,162)=11.5, p<.01]$. Group $\mathrm{LiCl}$ showed longer periods of lying on their bellies than did Group Saline during Min 12-27 (ps<.05).

\section{Discussion}

Lithium and amphetamine elicited some similar and some dissimilar URs. The grooming-related activities of body washing, face washing, and scratching were suppressed in rats experiencing either drug. However, linecrossing activity and rearing activity were suppressed in lithium-treated rats, but were enhanced in amphetamine-treated rats. Finally, the behaviors of body dragging and lying on bellies were seen only in lithiumtreated rats.

\section{GENERAL DISCUSSION}

The preceding experiments investigated the nature of behavioral CRs that were elicited by a lithium-paired or an amphetamine-paired contextual chamber CS and of behavioral URs that were elicited by lithium and amphetamine. The grooming-related activities of body 
washing, face washing, and/or scratching tended to be suppressed in all experiments. That is, lithium and amphetamine elicited suppression of grooming activities as URs, and this suppressed grooming was also apparent as a CR with both drug conditions. The suppression of grooming thus appears to represent a nonspecific effect of both drugs, which is conditionable to a contextual cue.

There were, however, a number of behaviors that differed among experiments, apparently reflecting drugspecific conditioning of US properties. The responses of line crossings and rearing were differentially affected across drug conditions. Lithium elicited the URs of suppressed line crossings and rearing, whereas amphetamine elicited the URs of enhanced line crossings and rearing. The US properties thus differed between the two drug conditions on these measures. However, the CRs did not reflect properties that were similar to the URs when the rats were exposed to the drug-paired contextual cues. Instead, the CRs reflected drug-opposite properties by the measures of rearing and/or line crossing. A lithium-paired context elicited enhanced rearing duration and an amphetamine-paired context elicited suppressed rearing and line-crossing frequency. Other investigators have reported that some CRs involve behavior changes that are opposite in direction from the URs (e.g., Siegel, 1975, 1979). Not only were drugopposite CRs evident, but also some CRs were apparent that were not evident as URs and some URs were apparent that were not evident as CRs. The lithium CR of enhanced limb flicking and the amphetamine CR of suppressed shaking were not evident as URs. Furthermore, the lithium URs of suppressed line crossings and face washing were not evident as CRs. Although the lithium URs of lying on bellies and body dragging were not explicitly measured as CRs, these behaviors were not obviously apparent during measurement of CRs.

A number of investigators have studied the form of the CR in Pavlovian conditioning (for a review, see Rescorla \& Holland, 1982). As the number of such investigations using different behavioral techniques increases, it has become apparent that, although the CR and the UR are frequently similar, they may also differ in various ways. Often the pattern of CRs does not include aspects of behavior that are evident in the pattern of URs elicited by the US (e.g., Zener, 1937), and sometimes the CR includes behaviors that are not part of the UR (e.g., Timberlake \& Grant, 1975; Zener, 1937). Furthermore, some CRs are evident as behaviors that are opposite in direction from the UR (e.g., Siegel, 1975). One must expect that when a wide range of behaviors are measured, some of each of these behavioral possibilities will occur, as was found here. Therefore, neither stimulus substitution theory nor theories that propose that the $C R$ represents a conditioned compensatory response elicited by the CS can fully account for our data. Instead, each mechanism may be responsible for different components of the CR pattern.
The form of the CR also often depends upon characteristics of the CS as well as upon those of the US. For example, Holland (1977) demonstrated that the pattern of CRs elicited by a food US is a function of the CS modality. Parker (1982) found that a flavor CS previously paired with either lithium or amphetamine elicited the common $\mathrm{CR}$ pattern of enhanced line crossings and rearing behavior; however, only lithiumpaired flavors elicited chin-rub behavior. None of the above experiments produced any evidence of chin-rub behavior (as CRs or URs), and, therefore, this behavior is probably observed only when flavors serve as the CS. Furthermore, a lithium-paired contextual cue elicited enhanced rearing, as in Parker (1982), but an amphetamine-paired contextual cue elicited suppressed line crossings and rearing during the first $5 \mathrm{~min}$ of testing. Therefore, the form of the drug CR appears to be influenced not only by the specific US employed, as in the present study, but also by the specific CS employed.

\section{REFERENCES}

Braveman, N. (1975). Formation of taste aversions in rats following prior exposure to sickness. Learning and Motivation, 6, 512-534.

GrILL, H., \& Norgren, R. Taste reactivity test I. Mimetic responses to gustatory stimuli in neurologically normal rats. Brain Research, 1978, 143, 263-279.

Holland, P. C. (1977). Conditioned stimulus as a determinant of the form of the Pavlovian conditioned response. Journal of Experimental Psychology: Animal Behavior Processes, 3, 77-104.

Johnson, F. N. (1979). The psychopharmacology of lithium. Neuroscience and Biobehavioral Reviews, 3, 15-30.

Lubow, R. E., \& Moone, A. U. (1959). Latent inhibition: The effect of nonreinforced preexposure to the conditioned stimulus. Journal of Comparative and Physiological Psychology, 52, 415-419.

Parker, L. A. (1982). Nonconsummatory and consummatory behavioral CRs elicited lithium- and amphetamine-paired flavors. Learning and Motivation, 13, 281-303.

Pavlov, I. P. (1927). Conditioned reflexes. London/New York: Oxford University Press.

PiCKens, R., \& Crowder, W. (1967). Effect of CS-US interval on conditioning of drug response, with assessment of speed of conditioning. Psychopharmacologia, 11, 88-94.

Rescorla, R., \& Holland, P. C. (1982). Behavioral studies of associative learning in animals. Annual Review of Psychology, 33, 265-308.

RILey, A., \& BArIL, L. (1976). Conditioned taste aversions: A bibliography. Animal Learning \& Behavior. 4, 1S-13S.

SIEGEL, S. (1975). Evidence from rats that morphine tolerance is a learned response. Joumal of Comparative and Physiological Psychology, 89, 498-506.

Sizard, S. (1979). Learning and psychopharmacology. In M. E. Jarvik (Ed.), Psychopharmacology in the practice of medicine. New York: Appleton-Century-Crofts.

Tilson, H. A., \& Rech, R. H. (1973). Conditioned drug effects and absence of tolerance to d-amphetamine induced motor activity. Pharmacology, Biochemistry and Behavior, 1, 149-153.

Timberlake, W., \& Grant, D. (1975). Autoshaping in rats to the presentation of another rat predicting food. Science, 190, 690-692.

ZENER, K. (1937). The significance of behavior accompanying salivary secretion for theories of the conditioned response. American Journal of Psychology, 50, 384-403.

(Manuscript received January 10, 1984; revision accepted for publication May 22, 1984.) 